\title{
Management of Major Neurocognitive Disorders in African-Americans
}

\author{
Carl C Bell ${ }^{1 *}$ and Jessie Aujla ${ }^{2}$ \\ ${ }^{1}$ Department of Psychiatry, School of Medicine, University of Illinois at Chicago, USA \\ ${ }^{2}$ Senior Medical Student at St. James medical school, USA
}

*Correspondence to: Carl C. Bell, Clinical Professor of Psychiatry Emeritus, Department of Psychiatry, School of Medicine, University of Illinois, Chicago, USA; Tel: +1 (773) 633-5450; Fax: +1 (773) 947-7721; E-mail: bell-carl@att.net

Received: September 15, 2017; Accepted: September 25, 2017; Published: September 25, 2017;

\begin{abstract}
This paper is a retrospective chart review study of African-American patients 55 years and older that were referred for psychiatric consultation after admission to a medical/surgical unit with integral psychiatric support at Jackson Park Hospital serving predominately African-Americans on Chicago's Southside. Thirty-two percent (39/121) were found to have Computerized Tomography scan brain pathology (cerebral atrophy, cerebral ischemia, or cerebral infarction). Based on clinical judgment, these patients and others who had clinical findings necessitating a diagnosis of Major Neurocognitive Disorder, with and without Computerized Tomography scan brain pathology, were given Selective Serotonin Reuptake Inhibitors (chiefly Escitalopram starting at $5 \mathrm{mg}$ every morning and increasing the dose until the patient responded rarely over $10 \mathrm{mg}$ ). Of these 39,29 were more agreeable and had brighter affects, although the Sensorium and Cognition of their mental status examinations did not change, four showed absolutely no change, and six were lost to follow up, but who were well enough to be psychiatrically cleared for discharge.
\end{abstract}

Key Words: African-American, Neurocognitive disorders, Computerized tomography, Escitalopram, Point-prevalence, Major Depressive Disorder (MDD)

\section{Introduction}

The rates of Major Neurocognitive Disorder (MNCD) (specifically Alzheimer's disease - AD) in African-Americans are reported to be twice as high as the rates in European-Americans. [1] The reasons for this health disparity are unknown, but that lack of understanding does not stop the influx of such patients to inpatient consultation and liaison services in general hospitals. Such patients are often referred for psychiatric evaluations from nursing homes because of aggressive behavior and a lack of cooperativeness. Unfortunately, the literature regarding African-American health and wellness is sparse and it is up to clinical research to fill this void until research that is more academic is available. [2] Further, clinical research often leads to the most pragmatic academic research. [3]

This brief report focuses on the prevalence and features of Neurocognitive Disorders in African-Americans admitted to Jackson Park Hospital - a hospital on Chicago's Southside serving a predominately low-income African-Population. Previous research on this patient population revealed $98 \%$ of the patients Jackson Park Hospital services resided in one of the three zip codes (60617, 60619, and 60649) on Chicago's South Side (Avalon Park, Burnside, Chatham, Greater Grand Crossing, and South Shore communities). [4] About 143,000 people live in these communities, and their median household income is $\$ 33,809$. The patients sampled were hospitalized between May 1, 2017 to August 31, 2017.

\section{Method}

We sampled all patients admitted to Jackson Park Hospital's Medical/Surgical - Psychiatric Inpatient unit from May 1 to August 31, 2017 who where 55 and up, and who were asked to have psychiatric consultations by the medical/surgical staff. The fore mentioned patients' medical records were reviewed retrospectively, carefully examined and it was checked if a Head Computed Tomography (CT) scan without contrast was performed, as well as if Escitalopram or another Selective Serotonin Reuptake Inhibitor (SSRI) was utilized as part of the patient's medical treatment plan. From there, the patient's medical notes were further explored; the Head CT scans were viewed and examined in order to determine if the image demonstrated any pathology, such as cerebral atrophy, infarction and/or ischemia. Other factors were considered: 1) anti-psychotic use 2) employment of different selective serotonin reuptake inhibitors 3 ) if the patient was admitted from a nursing home and 4) if the patient was previously admitted to Jackson Park Hospital. The first author then conducted psychiatric evaluations for patients seeking services at the inpatient unit. The patients were admitted due to many different complaints, from aggressive behavior/altered mental status to unspecified "Dementia" (replaced in DSM-5 by Major Neurocognitive Disorder). Based on past clinical experience patients who were suspected of having central nervous system damage were given Escitalopram 5 - $10 \mathrm{mg}$, as it was the author's clinical experience that patients with suspected central nervous system damage did well on this particular selective serotonin reuptake inhibitor. [5] Jackson Park Hospital's 
Institution Review board approved the project as the data was archival and all patient identifiers were stripped from their files.

\section{Results}

Of 147 patient's ( 55 years or older) records were reviewed but 26 subjects were lost to follow up leaving a total population of 121 patients over 55 . Of these, 50 had clinical indications to get CT scans without contrast and they were given Escitalopram starting out at $5 \mathrm{mg}$ and given up to $10 \mathrm{mg}$ depending on their over-all health. [5] Based on clinical judgment, 39 of the 121 patients (32.2\%) were given Escitalopram, usually starting out with $5 \mathrm{mg}$ every morning, and these patients showed CT pathology (cerebral atrophy, cerebral ischemia, or cerebral infarction). Of these 39, 29 were more agreeable and had brighter affect, although the Sensorium and Cognition of their mental status examinations did not change, four showed absolutely no change, and six were lost to follow up, but who were well enough to be psychiatrically cleared for discharge. Seven patients were ordered to have a CT scan but the CT scans were not available for review; of these, four improved in being agreeable and their affect was noticeably brighter, but not in mental status Sensorium or Cognition; and three stayed the same. Fourteen patients showed CT pathology but were not given SSRIs. There were eleven patients who did not get CT scans, but who were given Escitalopram. Ten patients demonstrated improvement with Escitalopram similar to the patients described above, while one patient was lost due to discharge by the weekend psychiatric coverage. Additionally, five patients were given Escitalopram but were lost to follow up as they were discharged. Seven other patients were administered other selective serotonin reuptake inhibitors, and two patients showed improvement with Sertraline and Fluoxetine; while the other five were lost due to being discharged or no follow-up was deemed warranted (Table 1).

Table 1.

\begin{tabular}{|c|c|c|c|c|c|c|c|c|}
\hline Initials & $\begin{array}{l}\text { Year of } \\
\text { Birth }\end{array}$ & $\begin{array}{l}\text { CT (Y/N, If Y=(NL), } \\
\text { (NA), (A), (IS), (IN) }\end{array}$ & $\begin{array}{c}\text { Lexap }(\mathrm{Y} / \mathrm{N}, \text { If } \mathrm{Y}=\text { progress - } \\
\text { other SSRI) }\end{array}$ & Diagnosis & Antipsychotic Use D/C & NH $(\mathbf{Y} / \mathbf{N})$ & Previous Admit & Race \\
\hline A.A. & 1959 & Yes $=\mathrm{A}, \mathrm{IS}$ & $\begin{array}{l}\text { Yes (Other Psychiatrist } \\
\text { cleared the patient) }\end{array}$ & Psychosis NOS & No & Yes & No & Black \\
\hline A.K. & 1938 & Yes $=$ NA & Yes(same) & MNCD & $\mathrm{D} / \mathrm{C}$ risperidone & Yes & Yes & Black \\
\hline A.N. & 1961 & Yes $=\mathrm{A}, \mathrm{IS}$ & NO NOTE AVAILABLE & & & & & Black \\
\hline A.S. & 1959 & Yes $=\mathrm{NL}$ & NO & MDD & $\begin{array}{l}\text { Yes (ziprasidone, } \\
\text { risperidone) }\end{array}$ & No & No & Black \\
\hline B.A. & 1961 & Yes $=\mathrm{NL}$ & No & Psychosis NOS & ziprasidone by resident & Yes & No & White \\
\hline B.A. & 1959 & No & No & $\mathrm{EtOH}$ & No & No & No & Black \\
\hline B.A. & 1961 & Yes $=\mathrm{A}, \mathrm{IS}$ & No & Depression NOS & No & No & No & Black \\
\hline B.B. & 1944 & Yes $=\mathrm{A}, \mathrm{IS}$ & $\begin{array}{l}\text { Yes (Improved on previous } \\
\text { admission) }\end{array}$ & MNCD & No & Yes & Yes & Latino \\
\hline B.B. & 1949 & Yes $=\mathrm{A}$ & No & Psychosis NOS & Yes (Haloperidol) & No & Yes & Black \\
\hline B.B. & 1957 & Yes $=\mathrm{NL}$ & No & $\begin{array}{l}\text { Deferred (then } \\
\text { other Psychiatrist) }\end{array}$ & Yes (Haloperidol) & No & No & Black \\
\hline B.B. & 1955 & Yes $=\mathrm{A}, \mathrm{IS}$ & Yes (Better) & MNCD & No & No & No & Black \\
\hline B.C. & 1960 & No & No & $\begin{array}{l}\text { Paranoid } \\
\text { Schizophrenia (not } \\
\text { enough history }\end{array}$ & Yes ( Loxapine0 & No & No & White \\
\hline B.D. & 1959 & Yes $=\mathrm{NL}$ & No & PTSD (by history) & No & No & No & Black \\
\hline B.J. & 1940 & No & No & Schizoaffective & Yes (risperidone) & Yes & No & Black \\
\hline B.J. & 1962 & No & No & $\mathrm{EtOH}$ & No & No & No & Black \\
\hline B.L. & 1946 & Yes $=\mathrm{NL}$ & No & $\begin{array}{l}\text { No Psychiatric } \\
\text { Diagnosis }\end{array}$ & No & No & No & Black \\
\hline B.R. & 1956 & No & No & $\begin{array}{l}\text { Intellectual } \\
\text { Disability }\end{array}$ & No & Yes & No & Black \\
\hline B.R. & 1932 & Yes $=\mathrm{A}$ & No & $\begin{array}{l}\text { MNCD (Other } \\
\text { Psychiatrist) }\end{array}$ & Yes (Quetiapine) & Yes & No & White \\
\hline B.T. & 1940 & Yes $=\mathrm{A}$, IS & No & Metastatic HPC & No & No & No & Black \\
\hline
\end{tabular}




\begin{tabular}{|c|c|c|c|c|c|c|c|c|}
\hline Initials & $\begin{array}{l}\text { Year of } \\
\text { Birth }\end{array}$ & $\begin{array}{l}\text { CT (Y/N, If Y=(NL), } \\
\text { (NA), (A), (IS), (IN) }\end{array}$ & $\begin{array}{c}\text { Lexap (Y/N, If } \mathrm{Y}=\text { progress - } \\
\text { other SSRI) }\end{array}$ & Diagnosis & Antipsychotic Use D/C & NH $(\mathbf{Y} / \mathbf{N})$ & Previous Admit & Race \\
\hline B.W. & 1951 & Yes $=\mathrm{A}, \mathrm{IS}$ & Yes (fine, no longer agitated) & MNCD & $\begin{array}{l}\text { Lurasidone by attending } \\
\text { physician }\end{array}$ & Yes & No & White \\
\hline B.W. & 1940 & No & Yes (calm) & Psychosis NOS & No & Yes & Yes & White \\
\hline C.L. & 1951 & Yes $=\mathrm{A}$ & Yes (calmer, less confused) & MNCD & $\begin{array}{l}\text { Quetiapine was } \\
\text { discontinued }\end{array}$ & Yes & No & Black \\
\hline C.L. & 1951 & Yes $=\mathrm{NA}$ & Yes (lowered, still lethargic) & MNCD & No & Yes & No & Black \\
\hline C.M. & 1958 & No & No & Depression NOS & Yes (risperidone) & No & No & Black \\
\hline C.P. & 1955 & No & $\begin{array}{l}\text { No (was given Paroxetine by } \\
\text { other Psychiatrist) }\end{array}$ & $\begin{array}{l}\text { Major Depression } \\
\text { by other } \\
\text { Psychiatrist }\end{array}$ & No & No & No & Black \\
\hline C.R. & 1961 & No & Yes (by other Psychiatrist) & $\begin{array}{l}\text { Major Depression } \\
\text { by other } \\
\text { Psychiatrist }\end{array}$ & No & No & No & Black \\
\hline C.S. & 1954 & Yes $=\mathrm{NL}$ & No & Adjustment & No & No & Yes & Black \\
\hline C.W. & 1939 & Yes $=$ NA & Yes (well) & Adjustment & No & Yes & No & Black \\
\hline D.B. & 1932 & Yes $=\mathrm{A}$, IS & $\begin{array}{l}\text { Yes ("brighter", brighter, } \\
\text { more energy) }\end{array}$ & MNCD & No & Yes & Yes & Black \\
\hline D.B. & 1958 & Yes $=\mathrm{NL}$ & No & $\begin{array}{l}\text { Intellectual } \\
\text { Disability }\end{array}$ & $\begin{array}{l}\text { Yes (Chloropromazine, } \\
\text { risperidone) }\end{array}$ & Yes & No & Black \\
\hline D.G. & 1951 & Yes $=$ IS, Volume Loss & Yes (better, brighter) & TBI & No & Yes & Yes & Black \\
\hline $\begin{array}{l}\text { D.J. } \\
\text { was } \\
\text { F.K. }\end{array}$ & 1954 & No & Yes ( better) & MNCD & No & No & No & Black \\
\hline D.L. & & & & DECEASED & & & & \\
\hline D.M. & 1952 & Yes $=\mathrm{A}, \mathrm{IS}$ & Yes (not talking) & $\mathrm{MNCD}$ & Haloperidol by Resident & No & No & Black \\
\hline D.P. & 1961 & No & No & Substance Abuse & No & No & Yes & Black \\
\hline D.S. & 1935 & Yes $=\mathrm{NL}$ & No & $\begin{array}{l}\text { Mood Disorder } \\
\text { NOS }\end{array}$ & Yes (Quetiapine) & Yes & No & Black \\
\hline D.w. & 1934 & Yes $=\mathrm{A}, \mathrm{IS}$ & Yes (Awake, better) & MNCD & $\mathrm{D} / \mathrm{C}$ risperidone & Yes & Yes & Black \\
\hline E.A. & 1936 & Yes $=\mathrm{A}, \mathrm{IS}$ & Yes ("pretty good") & MNCD & No & No & No & Black \\
\hline E.D. & 1951 & No & No & Acute Psychosis & $\begin{array}{l}\text { Quetiapine, ziprasidone } \\
\text { (changed all to HS and } \\
\text { D/C Benztropine) }\end{array}$ & No & No & Black \\
\hline E.E. & 1956 & Yes $=\mathrm{A}, \mathrm{IS}$ & Yes (little clamer) & $\mathrm{MNCD}$ & $\begin{array}{l}\text { Clozapine (given by } \\
\text { resident) }\end{array}$ & Yes & No & White \\
\hline E.G. & 1959 & Yes $=\mathrm{NL}$ & No & $\begin{array}{l}\text { Intellectual } \\
\text { Disability }\end{array}$ & $\begin{array}{l}\text { Quetiapine (given by } \\
\text { resident) }\end{array}$ & No & No & Black \\
\hline E.J. & 1935 & Yes $=\mathrm{A}, \mathrm{IS}$ & Yes (Discharged) & MNCD & No & Yes & No & Black \\
\hline F.H. & 1943 & Yes $=$ NA & No & $\begin{array}{l}\text { Schizophrenia by } \\
\text { other Psychiatrist, } \\
\text { Psychosis NOS }\end{array}$ & $\begin{array}{l}\text { Lower Benztropine, } \\
\text { risperidone by resident }\end{array}$ & Yes & Yes & Black \\
\hline G.B. & 1958 & Yes $=$ IS, IN & No & MDD & Loxapine & No & Yes & Black \\
\hline G.C. & 1947 & Yes $=A$, IS & Yes (better, hungry) & Depression NOS & D/C Quetiapine & Yes & No & White \\
\hline G.D. & 1937 & $\begin{array}{l}\text { Yes = Lacune Rt Basal } \\
\text { Ganglia }\end{array}$ & No & $\begin{array}{l}\text { Anxiety Disorder } \\
\text { NOS }\end{array}$ & No & No & No & Black \\
\hline G.E. & 1942 & Yes $=\mathrm{A}$ & $\begin{array}{l}\text { Yes (better. "think and } \\
\text { realize") }\end{array}$ & Psychosis NOS & No & No & Yes & Black \\
\hline
\end{tabular}




\begin{tabular}{|c|c|c|c|c|c|c|c|c|}
\hline Initials & $\begin{array}{l}\text { Year of } \\
\text { Birth }\end{array}$ & $\begin{array}{l}\text { CT (Y/N, If Y=(NL), } \\
\text { (NA), (A), (IS), (IN) }\end{array}$ & $\begin{array}{c}\text { Lexap (Y/N, If } \mathrm{Y}=\text { progress - } \\
\text { other SSRI) }\end{array}$ & Diagnosis & Antipsychotic Use D/C & NH $(\mathbf{Y} / \mathbf{N})$ & Previous Admit & Race \\
\hline G.G. & 1961 & Yes $=\mathrm{NL}$ & Yes (refused0 & $\begin{array}{l}\text { Intellectual } \\
\text { Disability }\end{array}$ & No & Yes & No & Black \\
\hline G.J. & 1950 & Yes $=$ IS & No & Adjustment & No & Yes & No & Black \\
\hline G.M. & 1941 & Yes $=\mathrm{A}, \mathrm{IS}$ & Yes (“fine”) & MNCD & No & Yes & No & Black \\
\hline G.R. & 1957 & ? SI, HI; psych consult & & & & & & \\
\hline G.T. & 1953 & Yes $=\mathrm{NL}$ & No & $\mathrm{EtOH}$ & No & No & Yes & Black \\
\hline G.V. & 1939 & Yes $=A$, IS, IN & No & Psychosis NOS & No & Yes & Yes & Black \\
\hline H.B. & 1955 & No & No & Schizophrenia & $\begin{array}{l}\text { Quetiapine (given by } \\
\text { resident) }\end{array}$ & Yes & No & Black \\
\hline H.C. & 1959 & No & No & Substance Abuse & No & No & No & Black \\
\hline H.D. & 1957 & No & Yes (sleeping well) & Anxiety, MDD & $\begin{array}{l}\text { Quetiapine (given by } \\
\text { resident) }\end{array}$ & No & No & Black \\
\hline H.E. & 1957 & No & $\begin{array}{l}\text { Yes (much more responsive, } \\
\text { whole new person) }\end{array}$ & $\begin{array}{l}\text { Organic Brain } \\
\text { Damage from } \\
\text { CVA }\end{array}$ & No & No & No & Black \\
\hline H.J. & 1958 & & & $\begin{array}{l}\text { Not available, } \\
\text { Patient refused }\end{array}$ & & & No & \\
\hline H.L. & 1952 & Yes $=$ IS & Yes (calmer) & Heroin Abuse & No & No & No & Black \\
\hline H.M. & 1954 & Yes $=\mathrm{IN}$ & No & $\mathrm{EtOH}$ & No & No & No & Black \\
\hline H.P. & 1952 & Yes $=$ NA & Yes (much better) & $\begin{array}{l}\text { Anxiety Disorder } \\
\text { NOS }\end{array}$ & No & Yes & No & Black \\
\hline H.R. & & CURRENT & & & & & & \\
\hline H.T. & 1954 & Yes $=\mathrm{NL}$ & No & Psychosis NOS & Loxapine & No & No & Black \\
\hline H.W. & 1931 & Yes $=\mathrm{A}, \mathrm{IS}$ & Yes (more responsive) & MNCD & $\begin{array}{l}\text { Olanzapine (given by } \\
\text { resident) }\end{array}$ & Yes & Yes & Black \\
\hline J.A. & 1944 & Yes $=\mathrm{A}$ & Yes & $\begin{array}{l}\text { MNCD (Other } \\
\text { Psychiatrist) }\end{array}$ & No & Yes & No & Black \\
\hline J.D. & 1940 & Yes $=\mathrm{A}, \mathrm{IS}$ & $\begin{array}{l}\text { Yes ( } \mathrm{D} / \mathrm{C} \text { Benzo, anxiety is } \\
\text { gone \& she's happy) }\end{array}$ & MNCD & No & Yes & No & Black \\
\hline J.L. & 1956 & Yes $=$ NA & $\begin{array}{l}\text { Yes (continues to refuse to } \\
\text { talk) }\end{array}$ & MNCD & No & Yes & Yes & Black \\
\hline J.W. & 1957 & Yes $=N L$ & Yes (oriented and reasonable) & TBI & $\begin{array}{l}\text { risperidone (given by } \\
\text { resident) }\end{array}$ & Yes & Yes & White \\
\hline K.N. & 1956 & $\begin{array}{l}\text { Yes }=\text { Severe Volume } \\
\text { Loss, IS }\end{array}$ & No & Adjustment & No & Yes & No & White \\
\hline K.W. & 1937 & Yes $=$ NA & Yes (well) & Anxiety & No & No & Yes & Black \\
\hline L.B. & 1950 & Yes $=\mathrm{A}, \mathrm{IS}$ & Yes (“fine") & MNCD & No & Yes & No & Black \\
\hline L.G. & 1961 & $\begin{array}{l}\text { Yes }=\text { consistent with } \\
\text { MS }\end{array}$ & Yes (same, calmer) & $\begin{array}{l}\text { Intellectual } \\
\text { Disability }\end{array}$ & No & Yes & No & Black \\
\hline L.L. & & CURRENT & & & & & & \\
\hline L.R. & 1936 & Yes $=\mathrm{A}$ & Yes (looks better) & Psychosis NOS & No & Yes & Yes & White \\
\hline M.A. & 1946 & Yes $=$ IS & No & Psychosis NOS & Loxapine & Yes & No & Black \\
\hline M.E. & 1960 & Yes $=$ NA & No & $\begin{array}{l}\text { Intellectual } \\
\text { Disability }\end{array}$ & risperidone by resident & Yes & No & Black \\
\hline M.F. & 1925 & Yes $=\mathrm{A}, \mathrm{IS}$ & $\begin{array}{l}\text { ????????? } \\
\text { Geriartric Physician }\end{array}$ & & & & & \\
\hline
\end{tabular}




\begin{tabular}{|c|c|c|c|c|c|c|c|c|}
\hline Initials & $\begin{array}{l}\text { Year of } \\
\text { Birth }\end{array}$ & $\begin{array}{l}\text { CT (Y/N, If Y=(NL), } \\
\text { (NA), (A), (IS), (IN) }\end{array}$ & $\begin{array}{c}\text { Lexap (Y/N, If } \mathrm{Y}=\text { progress - } \\
\text { other SSRI) }\end{array}$ & Diagnosis & Antipsychotic Use D/C & NH (Y/N) & Previous Admit & Race \\
\hline M.H. & 1959 & Yes $=\mathrm{A}, \mathrm{IS}$ & $\begin{array}{l}\text { Yes (better, more alert, not } \\
\text { aggressive) }\end{array}$ & MNCD (unknown) & risperidone was lowered & Yes & Yes & Black \\
\hline M.J. & 1944 & Yes $=\mathrm{A}$ & Yes (mood is okay) & $\begin{array}{l}\text { Intellectual } \\
\text { Disability }\end{array}$ & No & No & No & Black \\
\hline M.K. & 1945 & Yes $=$ NL & No (Mirtazapine) & $\begin{array}{l}\text { Major Depression } \\
\text { by other } \\
\text { Psychiatrist }\end{array}$ & & No & No & Black \\
\hline M.L. & 1957 & No & No & $\mathrm{EtOH}$ & No & No & No & Black \\
\hline M.M. & 1961 & Yes $=$ NA & No (Sertraline) & Depression NOS & Quetiapine & No & Yes & Black \\
\hline M.R. & 1961 & Yes $=\mathrm{A}$ & No & MDD, TBI & Quetiapine & No & Yes & White \\
\hline M.R. & 1955 & Yes $=\mathrm{IS}, \mathrm{A}$ & Yes (Alert) & Organic or MNCD & No & No & No & Black \\
\hline M.R. & 1947 & Yes $=$ NA & No & Psychosis NOS & Olanzapine by resident & Yes & Yes & Latino \\
\hline M.S. & 1956 & $\begin{array}{l}\text { Yes }= \\
\text { Encaphalomalacia }\end{array}$ & No & $\begin{array}{l}\text { Intellectual } \\
\text { Disability, TBI }\end{array}$ & No & No & Yes & Black \\
\hline M.V. & 1952 & No & No & Psychosis NOS & No & No & No & Black \\
\hline M.W. & 1957 & Yes $=$ NA & No & $\mathrm{EtOH}$ & No & No & No & Black \\
\hline M.W. & 1960 & Yes $=\mathrm{A}, \mathrm{IN}$ & Yes (Alert, responsive) & $\begin{array}{l}\text { Organic Brain } \\
\text { Damage }\end{array}$ & No & No & No & Black \\
\hline N.C. & 1962 & No & No & $\begin{array}{l}\text { Major Depression } \\
\text { by other } \\
\text { Psychiatrist }\end{array}$ & & No & No & Black \\
\hline N.E. & 1949 & No & $\begin{array}{l}\text { Yes (better, thinking is much } \\
\text { clearer) }\end{array}$ & MNCD & No & No & No & Black \\
\hline N.J. & 1939 & Yes $=\mathrm{A}, \mathrm{IS}$ & Yes (little better) & MNCD & Haloperidol by Resident & Yes & No & Black \\
\hline N.Z. & 1954 & Yes $=\mathrm{A}, \mathrm{IS}$ & & $\begin{array}{l}\text { ????? Chief } \\
\text { complaint was } \\
\text { acute psychosis, } \\
\text { an attending } \\
\text { physician patient }\end{array}$ & & Yes & No & \\
\hline O.E. & 1949 & No & No (Paroxetine by resident) & TBI & No & Yes & No & White \\
\hline O.F. & 1961 & No & No & Psychosis NOS & Loxapine, $\mathrm{d} / \mathrm{c}$ Olanzapine & No & No & Black \\
\hline O.J. & 1961 & No & No & Acute Psychosis & Loxapine & Yes & No & White \\
\hline O.N. & 1946 & Yes $=\mathrm{A}, \mathrm{IS}$ & Yes (“allright”, better) & $\mathrm{MNCD}$ & No & Yes & Yes & Black \\
\hline O.V. & 1956 & Yes $=$ NA & Yes (did not get it) & Depression NOS & Continue Quetiapine & No & Yes & Black \\
\hline P.D. & 1940 & Yes $=\mathrm{A}$ & Yes (no different) & $\mathrm{MNCD}$ & No & Yes & Yes & White \\
\hline P.H. & 1956 & Yes $=$ IS & No & Heroin, MDD & No & No & Yes & Black \\
\hline P.J. & 1951 & No & No (fluoxetine) & MDD & ziprasidone by resident & Yes & No & White \\
\hline P.J. & 1937 & Yes $=\mathrm{IN}$ & No & Psychosis NOS & Loxapine & Yes & No & White \\
\hline P.M. & 1938 & Yes $=A$, IS, IN & $\begin{array}{l}\text { Yes (other Psychiatrist } \\
\text { cleared pt, "calm and } \\
\text { coherent, no agitation or } \\
\text { behavioral problems" }\end{array}$ & $\mathrm{MNCD}$ & No & Yes & No & Black \\
\hline P.N. & 1948 & Yes $=$ IS & No & Psychosis NOS & Loxapine refused & Yes & No & Black \\
\hline P.R. & 1952 & Yes $=N L$ & Yes (little better, flirts) & Psychosis NOS & risperidone & Yes & Yes & Black \\
\hline R.B. & 1937 & Yes $=\mathrm{A}$, IS & Yes (better) & MNCD & No & Yes & Yes & White \\
\hline
\end{tabular}




\begin{tabular}{|c|c|c|c|c|c|c|c|c|}
\hline Initials & $\begin{array}{l}\text { Year of } \\
\text { Birth }\end{array}$ & $\begin{array}{l}\text { CT (Y/N, If Y=(NL), } \\
\text { (NA), (A), (IS), (IN) }\end{array}$ & $\begin{array}{c}\text { Lexap (Y/N, If } \mathrm{Y}=\text { progress - } \\
\text { other SSRI) }\end{array}$ & Diagnosis & Antipsychotic Use D/C & NH $(\mathbf{Y} / \mathbf{N})$ & Previous Admit & Race \\
\hline R.B. & 1948 & No & & $\begin{array}{l}\text { Schizophrenia by } \\
\text { other Psychiatrist }\end{array}$ & Quetiapine by resident & Yes & No & Black \\
\hline R.C. & 1948 & $\begin{array}{l}\text { Yes }=\text { Hemorrhage } \\
\text { in } 2008\end{array}$ & No & $\begin{array}{l}\text { MNCD secondary } \\
\text { to CVA (BASED } \\
\text { ON PREVIOUS } \\
\text { ADMISSION }\end{array}$ & No & Yes & Yes & Black \\
\hline R.D. & 1953 & No & No & $\begin{array}{l}\text { EtOH, Paranoid } \\
\text { Schizophrenia }\end{array}$ & $\begin{array}{l}\text { Quetiapine given by } \\
\text { resident }\end{array}$ & No & Yes & Black \\
\hline R.F. & 1953 & Yes $=\mathrm{A}, \mathrm{IS}$ & Yes (no progress note) & Organic/TBI & D/C Quetiapine & Yes & Yes & P Rican \\
\hline R.I. & 1947 & Yes $=A$, IS, IN & $\begin{array}{l}\text { Yes (same, then increased to } \\
10 \mathrm{mg} \text {, then lost) }\end{array}$ & $\begin{array}{l}\text { MDD, Rule out } \\
\text { MNCD }\end{array}$ & No & Yes & Yes & Black \\
\hline R.J. & 1953 & Yes $=$ NA & $\begin{array}{l}\text { Yes (calmer, not as } \\
\text { rambuctious) }\end{array}$ & $\begin{array}{l}\text { Intellectual } \\
\text { Disabilty, } \\
\text { Depression NOS }\end{array}$ & No & No & Yes & Black \\
\hline R.J. & 1955 & Yes $=\mathrm{A}, \mathrm{IS}$ & Yes (LOST) & $\mathrm{MNCD}$ & & No & No & Black \\
\hline R.J. & 1930 & No & $\begin{array}{l}\text { Yes (Prior admision was } \\
\text { effective) }\end{array}$ & MNCD & No & Yes & No & Black \\
\hline R.S. & 1943 & Yes $=$ NL in 2011 & No & $\begin{array}{l}\text { Intellectual } \\
\text { Disability }\end{array}$ & Quetiapine by attending & No & No & Black \\
\hline S.E. & 1948 & Yes $=\mathrm{NL}$ & No & $\begin{array}{l}\text { MNCD secondary } \\
\text { to CVA }\end{array}$ & No & No & No & Black \\
\hline S.G. & 1958 & No & No & $\begin{array}{l}\text { Major Depression } \\
\text { by other } \\
\text { Psychiatrist }\end{array}$ & No & No & No & Black \\
\hline S.G. & 1961 & Yes $=$ NA & No & $\begin{array}{l}\text { Mood Disorder } \\
\text { NOS }\end{array}$ & $\begin{array}{l}\text { risperidone, Loxapine, } \\
\text { Benztropine by resident }\end{array}$ & Yes & Yes & White \\
\hline S.M. & 1959 & Yes $=$ NA & No & Epilepsy & Haloperidol Deconoate & No & Yes & Black \\
\hline S.R. & 1960 & No & Yes (little better, less voices) & Depression NOS & Haloperidol by Psychiatrist & No & No & Black \\
\hline S.R. & 1952 & & & $\begin{array}{l}\text { Schizoaffectve (by } \\
\text { other Psychiatrist) }\end{array}$ & & & & \\
\hline S.R. & 1947 & Yes $=$ NL in 2008 & $\begin{array}{l}\text { No (Sertraline by resident, } \\
\text { discharged) }\end{array}$ & Depression NOS & Quetiapine by resident & No & Yes & Black \\
\hline S.S. & 1957 & Yes $=\mathrm{A}, \mathrm{IS}$ & $\begin{array}{l}\text { Yes ("well", polite but still } \\
\text { delusional) }\end{array}$ & Psychosis NOS & $\begin{array}{l}\mathrm{D} / \mathrm{C} \text { risperidone, Added } \\
\text { Loxapine }\end{array}$ & Yes & No & Black \\
\hline S.S. & 1949 & Yes $=A, I S, I N$ & No & $\begin{array}{l}\text { Adjustment } \\
\text { Disorder NOS }\end{array}$ & No & Yes & No & Black \\
\hline S.T. & 1958 & No & No & Adjustment & No & Yes & No & White \\
\hline T.A. & 1955 & No & No & $\mathrm{EtOH}$ & Quetiapine & No & Yes & Black \\
\hline T.C. & 1959 & Yes $=$ NL in 2012 & No (fluoxetine by resident) & $\begin{array}{l}\text { Mood Disorder } \\
\text { NOS }\end{array}$ & No & No & No & White \\
\hline T.G. & 1955 & No & $\begin{array}{l}\text { Yes (no voices, no } \\
\text { aggression) }\end{array}$ & Acute Psychosis & $\begin{array}{l}\text { Lowered Benztropine, } \\
\text { Loxapine }\end{array}$ & Yes & No & Black \\
\hline T.J. & 1948 & Yes $=$ NA & No (was stable) & $\begin{array}{l}\text { Paranoid } \\
\text { Schizophrenia }\end{array}$ & No & Yes & Yes & Black \\
\hline T.P. & 1959 & Yes $=\mathrm{IN}$ & No & $\mathrm{EtOH}$ & No & No & No & Black \\
\hline T.R. & 1962 & No & No & Depression NOS & risperidone by resident & Yes & No & Black \\
\hline T.R. & 1953 & Yes $=\mathrm{A}, \mathrm{IS}$ & $\begin{array}{l}\text { Yes (slightly better, more } \\
\text { alert) }\end{array}$ & Psychosis NOS & No & No & No & White \\
\hline
\end{tabular}




\begin{tabular}{|c|c|c|c|c|c|c|c|c|}
\hline Initials & $\begin{array}{l}\text { Year of } \\
\text { Birth }\end{array}$ & $\begin{array}{l}\text { CT (Y/N, If Y=(NL), } \\
\text { (NA), (A), (IS), (IN) }\end{array}$ & $\begin{array}{c}\text { Lexap (Y/N, If } \mathrm{Y}=\text { progress - } \\
\text { other SSRI) }\end{array}$ & Diagnosis & Antipsychotic Use D/C & NH $(\mathbf{Y} / \mathbf{N})$ & Previous Admit & Race \\
\hline T.R. & 1958 & No & No & Epilepsy & Loxapine & No & No & Black \\
\hline T.R. & 1953 & & & $\begin{array}{l}\text { Decreased, } \\
\text { no psychiaric } \\
\text { diagnosis, Guillain } \\
\text { Bairre }\end{array}$ & & & & \\
\hline V.A. & 1957 & & & $\begin{array}{l}\text { Other Psychiatrist } \\
\text { (In April for } \\
\text { EtOH) }\end{array}$ & & No & Yes & \\
\hline W.A. & 1958 & $?$ & NO NOTE AVAILABLE & $\begin{array}{l}\text { Consult was } \\
\text { ordered, EtOH }\end{array}$ & & & & \\
\hline W.B. & 1958 & Yes $=\mathrm{A}, \mathrm{IS}$ & No (fluoxetine, much better) & Heroin & No & No & Yes & Black \\
\hline W.C. & 1946 & No & $\begin{array}{l}\text { Yes (much less cranky and } \\
\text { was pleasant) }\end{array}$ & MNCD & No & Yes & No & Black \\
\hline W.D. & 1957 & & No & $\begin{array}{l}\text { Intellectual } \\
\text { Disability }\end{array}$ & No & No & No & Black \\
\hline W.K. & 1956 & & & Other Psychiatrist & & & & \\
\hline W.L. & 1951 & $?$ & & & & & & \\
\hline W.M. & 1928 & Yes $=A$, IS, IN & Yes (lethargic) & MNCD & No & Yes & No & Black \\
\hline W.P. & 1953 & No & Yes (affect brighter) & Psychosis NOS & No & Yes & No & Black \\
\hline W.R. & 1962 & No & Yes (better, clamer) & Anxiety & No & No & No & Black \\
\hline W.R. & 1948 & Yes $=A$, IS, IN & Yes (same) & MNCD, EtOH & Quetiapine by resident & Yes & No & White \\
\hline W.T. & 1961 & Yes $=\mathrm{A}$ & Yes (better) & MNCD & Quetiapine by resident & Yes & Yes & White \\
\hline \multicolumn{9}{|c|}{ Total Subjects $=147$} \\
\hline \multicolumn{9}{|c|}{ Lost Subjects $=26$} \\
\hline \multicolumn{9}{|c|}{ CT performed and given Escitalopram $=50$, } \\
\hline \multicolumn{9}{|c|}{ CT path \& given Escitalopram $=39 ; 29$ showed improvement } \\
\hline \multicolumn{9}{|c|}{ CT path, given Escitalopram \& showed no change $=4$} \\
\hline \multicolumn{9}{|c|}{ CT path, given Escitalopram, but were lost $=6$} \\
\hline \multicolumn{9}{|c|}{ CT was ordered but not available and given Escitalopram $=7$ ( 4 with improvement, 3 were the same } \\
\hline \multicolumn{9}{|c|}{ CT with pathology but were not given Escitalopram or any type of SSRI $=14$} \\
\hline \multicolumn{9}{|c|}{ Subject did not undergo CT but was given Escitalopram $=10$} \\
\hline \multicolumn{9}{|c|}{$\mathrm{CT}(\mathrm{Y} / \mathrm{N}$, If $\mathrm{Y}=\operatorname{Normal}(\mathrm{NL})$} \\
\hline \multicolumn{9}{|c|}{ Not Available (NA) } \\
\hline \multicolumn{9}{|c|}{ Atrophy (A) } \\
\hline \multicolumn{9}{|c|}{ Ischemia (IS) } \\
\hline Infarctio & $(\mathrm{IN})$ & & & & & & & \\
\hline
\end{tabular}




\section{Discussion}

Serious scientific research has failed to show any serious prevention strategies for Alzheimer's disease. [6] However, as previously mentioned, African-Americans have been reported to have twice the Alzheimer's disease as European-Americans. [1] Apolipoprotein E is a risk allele for late onset Alzheimer's disease and compared with Caucasians, the allele frequency is higher among African Americans, and thus thought to be a significant risk factor for the development of Alzheimer's disease. [7] Unfortunately, as this was a retrospective study, the authors did not have a measure of the APOE genotype of all the study participants. Point prevalence studies on the prevalence of coma in African-American populations reveals high rates of coma in this population. [8] Having a loss of consciousness resulting in a coma from a traumatic brain injury is another risk factor for the development of Alzheimer's disease that is of interest to researchers, but which has yet to show a conclusive link between the two. [9] Other studies have shown that Alcohol-related Organic Brain Syndromes have been predisposed to misdiagnosis. [10] A review of the literature on coma illustrates this phenomena is frequently found in African-American communities and was hypothesized to contribute to the high rates of violence seen in such communities. [11-13] Another study that is more recent has revealed a high prevalence of Neurobehavioral Disorders associated with Prenatal Alcohol Exposure (ND-PAE) are found in poor African-American communities. [4] Accordingly, nearly $1 / 3$ of this predominately low-income African-American population having a Major Neurocognitive Disorder from cerebral atrophy, cerebral ischemia, or cerebral infarction is not surprising. However, these patients' extremely positive response to Escitalopram is surprising. Past experience with patients who had Alzheimer's disease, Cerebrovascular infarcts, or traumatic brain injuries leads one to believe there is not much that can be done for such patients. Escitalopram and other SSRIs do not improve their cognition. They reduce the patient's anxiety helping them to be less of a management problem for staff, while, at the same time, not leaving the patient lethargic and sleepy. Most times these patients' affects were noticeably brighter and they were more socially engaged despite not knowing the year or the president.

Much to the author's astonishment selective serotonin reuptake inhibitors (SSRIs) have been shown to reduce amyloidal deposits in brain, [14] and they do more to help patients with Major Neurocognitive Disorders than the usual donepezil or memantine which are seen as an unnecessary waste. It may well be that SSRI's are more effective at reducing and preventing amyloid formation in the damaged brain than the medications that have been approved for treating Alzheimer's disease. As the SSRI's are being prescribed for the patient's anxiety, they are not being used off label.

Another interesting long-term prevention strategy involved the use of prenatal choline. There is some indication that giving choline to pregnant women may prevent the development of Alzheimer's disease as well as other neurodevelopmental disorders. [15, 16] The schizophrenia research group at the University of Denver is recommending prenatal choline to prevent schizophrenia, autism and ADHD. [17, 18] Recently, the American Medical Association's House of Delegates provided advocacy to included recommended adequate doses of choline during pregnancy. [19] As of yet, there are no solid links between the high rates of ND-PAE and the high rates of Alzheimer's disease in African-Americans, but by virtue of the reality that adequate doses of prenatal choline are protective against both neuropsychiatric disorders, it would not surprise us.

\section{Conclusions}

Elderly African-American patients (55 years and older) referred for psychiatric consultation on a medical/surgical unit with psychiatry as an integral part of the treatment team often have cerebral pathology resulting in a diagnosis of Major Neurocognitive Disorders. In many instances the cerebral pathology can be documented by a CT scan without contrast. Although the congitive and sensorium of such patients is not significantly altered with an SSRI (most notably Escitalopram), there is a vast improvement in these patient's anxiety resulting in brighter affect and more agreeable behavior.

\section{References}

1. Alzheimer's Association (2014) African Americans and Alzheimer's disease: The Silent Epidemic. Chicago: USA

2. Mental Health: Culture, Race, and Ethnicity: A Supplement to Mental Health (2001): A Report of the Surgeon General. Rockville, MD: US Department of Health and Human Services, US Public Health Service.

3. Bell CC (2016) Commentary on the Usefulness of Clinical Research. Abnormal and Behavioural Psychology 2.

4. Bell CC, Chimata R (2015) Prevalence of Neurodevelopmental Disorders in LowIncome African-Americans at a Family Medicine Clinic on Chicago's Southside. Psychiatr Serv 66: 539-542. [Crossref]

5. Bell CC (2015) Managing major neurocognitive disorder in African Americans, Clinical Psychiatry News 43: 16.

6. Daviglus ML, Bell CC, Berrettini W, Bowen PE, et al. (2010) NIH State-of-theScience Conference: Preventing Alzheimer's disease and Cognitive Decline. Ann Intern Med 152: 176-181.

7. Yu L, Lutz MW, Wilson RS, Burns DK, et al. (2017) APOE e4- TOMM40 '523 haplotypes and the risk of Alzheimer's disease in older Caucasian and African Americans. PLoS ONE 12: e0180356. [Crossref]

8. Bell CC, Thompson B, Shorter-Gooden K, Shakoor B, Dew D, et al. (1985) Prevalence of coma in black subjects. J Natl Med Assoc 77: 391-395. [Crossref]

9. Julien J, Joubert S, Ferland MC, Frenette LC, Boudreau-Duhaime MM, et al. (2017) Association of traumatic brain injury and Alzheimer disease onset: A systematic review. Ann Phys Rehabil Med 60: 347-356. [Crossref]

10. Bell CC (1985) Alcohol-Related Organic Brain Syndromes-Frequently Misdiagnosed as Schizophrenia. Bulletin of the New York State Chapter of the National Black Alcoholism Council, Inc 4: 3-4.

11. Bell CC (1986) Coma and the etiology of violence, Part 1. J Natl Med Assoc 78: 1167-1176. [Crossref]

12. Bell CC (1987) Coma and the etiology of violence, Part 2. J Natl Med Assoc 79: 79-85. [Crossref]

13. Bell CC, Kelly R (1987) Head Injury with Subsequent Intermittent, Nonschizophrenic, Psychotic Symptoms and Violence. J Natl Med Assn 79: 1139-1144. [Crossref]

14. Sheline YI, West T, Yarasheski K, Swarm R et al. (2014) An Antidepressant Decreases CSF Aß Production in Healthy Individuals and in Transgenic AD Mice. Sci Transl Med 6: 236re4. [Crossref]

15. Bell CC (2017) Can prenatal choline lead to prevention of Alzheimer's? Clinical Psychiatry News 45 : 10-11. 
16. Strupp BJ, Powers BE, Velazquez R, Ash JA, et al. (2016) Maternal Choline Supplementation: A Potential Prenatal Treatment for Down Syndrome and Alzheimer's Disease. Curr Alzheimer Res 13: 97-106. [Crossref]

17. Freedman R1, Ross RG1 (2015) Prenatal choline and the development of schizophrenia. Shanghai Arch Psychiatry 27: 90-102. [Crossref]
18. Ross RG, Hunter SK, Hoffman MC, McCarthy L, et al. (2016) Perinatal Phosphatidylcholine Supplementation and Early Childhood Behavior Problems: Evidence for CHRNA7 Moderation. Am J Psychiatry 173: 209-516. [Crossref]

19. Bell CC (2017) AMA's stance on choline, prenatal vitamins could bring staggering results. Clinical Psychiatry News.

Citation:

Carl C Bell and Jessie Aujla (2017) Management of Major Neurocognitive Disorders in African-Americans. Ageing Sci Ment Health Stud Volume 1(1): 1-9 